\title{
Synthesis and anti-bacterial activities of novel 5-isonicotinoyl-1,2,3,4-tetrahydropyrimidines and bis-(5-isonicotinoyl-1,2,3,4-tetrahydropyrimidines)
}

\author{
J. N. Vishwakarma, ${ }^{\text {a* }}$ M. C. Dutta, ${ }^{a}$ K. Chanda, ${ }^{\text {a }}$ Bhaskar Das, ${ }^{\text {b }}$ M. A. Laskar, ${ }^{b}$ \\ and R. L. Nongkhlaw \\ ${ }^{a}$ Organic Research Lab., Department of Chemistry, St. Anthony’s College, Shillong- 793001 , \\ India \\ ${ }^{b}$ Department of Biotechnology, St. Anthony's College, Shillong-793 001, India \\ ${ }^{c}$ Department of Chemistry, North-Eastern Hill University, Shillong-793 022, India \\ E-mail: jnvishwakarma@,rediffmail.com
}

\begin{abstract}
Novel 5-isonicotinoyl-1,2,3,4-tetrahydropyrimidines 4a-i have been synthesized in good yields by the reaction of enaminones 3a-c, primary amines and formaldehyde. 4a-i have been screened for their antibacterial properties. Enaminones 3a-c have also been reacted with diamines and formaldehyde to give hitherto unreported bis-tetrahydropyrimidines 5a-l in good yields. The structures of 4a-i and 5a-I have been established with the help of spectral and analytical data.
\end{abstract}

Keywords: 4-Acetylpyridine, tetrahydropyrimidines, isonicotinoyl, bis- tetrahydropyrimidines

\section{Introduction}

Prompted by the biological activities of 5-nitro-1,2,3,4-tetrahydropyrimidine derivatives (NitroTHP-1) ${ }^{1} \&$ (Nitro-THP-2), ${ }^{2}$ we have recently reported facile synthetic routes to novel 5-benzoyl6-methylthio-1,2,3,4-tetrahydropyrimidines (Benzoyl-THP-3), ${ }^{3}$

5-benzoyl-1,2,3,4tetrahydropyrimidines (Benzoyl-THP-4) ${ }^{4}$ and bis-(5-benzoyl-1,2,3,4-tetrahydropyrimidines) (Benzoyl-THP-5) ${ }^{5}$ replacing the nitro group of position 5 of the tetrahydropyrimidine (THP) rings by benzoyl groups.<smiles>CN1CC([N+](=O)[O-])=C(NCc2ccc(Cl)nc2)N(C)C1</smiles>

Nitro-THP-1

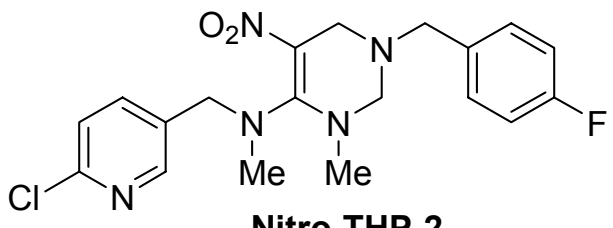

Nitro-THP-2 

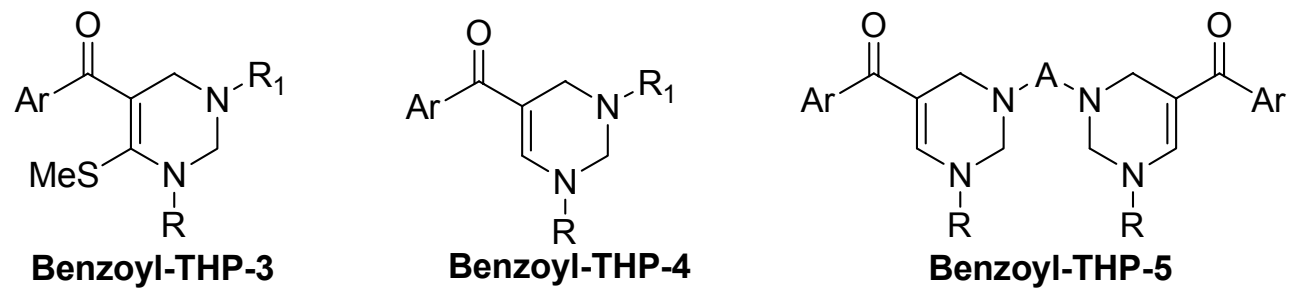

We envisaged that presence of another electron withdrawing group in position 5 of the tetrahydropyrimidine ring could have an important impact on the biological activities of these molecules. Our literature survey at this stage revealed that 1,2,3,4-tetrahydropyrimidines and bis(1,2,3,4-tetrahydropyrimidines) bearing isonicotinoyl group in position 5 of the ring are unknown in the literature to the best our knowledge and hence their biological properties remain unexplored. Furthermore, bis-compounds have received considerable importance as being model for main chain polymers. ${ }^{6-10}$ It is also reported that many biologically active natural and synthetic products have symmetry. ${ }^{11}$ As a result, many research groups are turning their attention to the synthesis of bis-heterocycles. ${ }^{12-15}$ Prompted by the promising biological properties of the tetrahydropyrimidines and hence bis-tetrahydropyrimidines bearing electron-withdrawing groups in position 5 of the ring, we decided to develop synthetic methodologies for 5-isonicotinoyl1,2,3,4-tetrahydropyrimidines and bis-(5-isonicotinoyl-1,2,3,4-tetrahydropyrimidines) and the results of our studies are reported herein. Antibacterial properties of 5-isonicotinoyl-1,2,3,4tetrahydropyrimidine derivatives have been studied and the results are presented in this paper.

\section{Results and Discussion}

In order to synthesize the proposed THPs bearing isonicotinyl group in position 5, enaminones of the type 3 derived from 4-acetylpyridine were required. Their synthesis could be achieved by first reacting 4-acetylpyridine with DMF-DMA following a previously reported procedure ${ }^{16}$ to yield the formylated product 2 and then converting 2 into 3 by a procedure ${ }^{17}$ developed in our laboratory. The structures of $\mathbf{3}$ as 3 -(phenyl/benzyl/methyl)amino-1-isonicotinoylpropenones were established with help of spectral and analytical data (experimental section). The enaminones 3a-c exist exclusively in Z-form as indicated by the highly deshielded N-H proton (10.76-12.25 ppm) signals due to hydrogen bonding and the low coupling constants of the vinylic protons $(\mathrm{J}=6 \mathrm{~Hz})$.

Synthesis of the desired tetrahydropyrimidines was subsequently undertaken. Thus when a mixture of enaminone 3a, methylamine and formaldehyde (1:1:2) in methanol was heated at reflux, work up of the reaction mixture gave $4 \mathbf{a}$ in $80 \%$ yields, the structure of which was proposed to be (1,2,3,4-Tetrahydro-3-methyl-1-phenylpyrimidin-5-yl)(pyridin-4-yl)methanone on the basis of spectral and analytical data. The reaction of $\mathbf{3 a}$ with other primary amines (benzyl amine or aniline) and formaldehyde proceeded under similar conditions giving the respective tetrahydropyrimidines $\mathbf{4 b}$ and $\mathbf{4} \mathbf{c}$ in $83 \%$ and $85 \%$ yields respectively. Similarly, the reaction of 
3b-c with appropriate primary amines (Scheme 1) and formaldehyde proceeded smoothly, giving the respective products $\mathbf{4 d - i}$ in good yields.

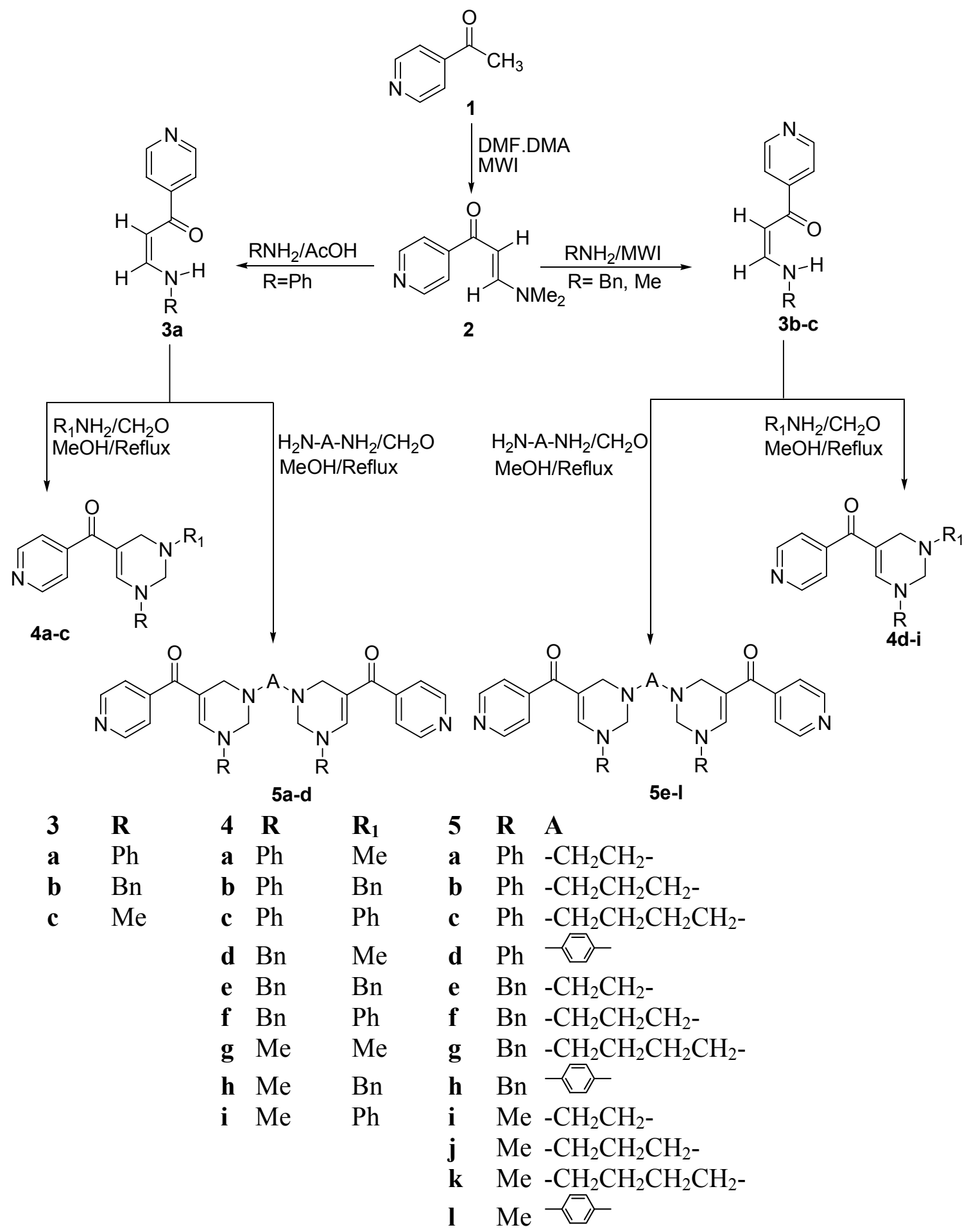

Scheme 1 
The infrared spectra of $\mathbf{4 a - i}$ showed strong peaks in the region of 1500 to $1630 \mathrm{~cm}^{-1}$ due to extensively delocalised double bonds and carbonyl groups. In the ${ }^{1} \mathrm{H} \mathrm{nmr}$ spectra of $\mathbf{4 a - i}$, the $\alpha$ and $\beta$ protons of pyridine ring appear as doublets in the vicinity of 8.60 and $7.30 \mathrm{ppm}$ respectively. The signal of $\mathrm{C}_{6}-\mathrm{H}$ proton of the THP ring remains obscured by the aromatic protons except in case of $\mathbf{4 g}$, where it was found resonating at $6.97 \mathrm{ppm}$. The signals due to benzylic $\mathrm{CH}_{2}$ protons in $\mathbf{4 b}$, $\mathbf{4 d - f}$ and $\mathbf{4 h}$ were found in the range of 3.65-3.90 ppm. The protons at $\mathrm{C}-4$ of the THP ring resonated just below $4.00 \mathrm{ppm}$ except in cases where phenyl group is attached to $\mathrm{N}-3$, while those bonded to $\mathrm{C}-2$ were more de-shielded and resonated in the vicinity of $4.50 \mathrm{ppm}$. In the ${ }^{13} \mathrm{C} \mathrm{nmr}$ spectra of the THPs, the most striking signal was due to carbonyl carbon (close to $190 \mathrm{ppm}$ ).

Subsequently, the reaction of $\mathbf{3}$ with diamines and formaldehyde was examined. Thus, when 3a $(2 \mathrm{mmol})$ was reacted with a mixture of ethylenediamine $(1 \mathrm{mmol})$ and formaldehyde (4 $\mathrm{mmol}$ ) in methanol, expected product 5a was obtained in 55\% yield, the structure of which was established to be [3,3'-(ethane-1,2-diyl)bis(1-phenyl-1,2,3,4-tetrahydropyrimidine-5,3diyl)]bis(pyridin-4-ylmethanone) based on spectral and analytical data. The reaction of $\mathbf{3 a}$ with other diamines $\left(\mathrm{A}=-\mathrm{CH}_{2} \mathrm{CH}_{2} \mathrm{CH}_{2}-,-\mathrm{CH}_{2} \mathrm{CH}_{2} \mathrm{CH}_{2} \mathrm{CH}_{2^{-}},-\mathrm{C}_{6} \mathrm{H}_{4}-\right)$ and formaldehyde led to the formation of the respective products $\mathbf{5 b - d}$ in $60-65 \%$ yields. Likewise, $\mathbf{3 b}$ and $\mathbf{3 c}$ reacted with diamines and formaldehyde under similar conditions giving the expected products 5e-i in good yields. The $\mathrm{N}-\mathrm{CH}_{2}$ protons of the linker chain of 5a-c, e-g, i-k resonated between 2.40 to 2.80 ppm, while other $\mathrm{CH}_{2}$ protons of the linker chains appeared as multiplets close to $1.75 \mathrm{ppm}$. The protons of the aromatic linkers remained obscured by other aromatic proton signals in their usual range.

\section{Anti-bacterial properties of tetrahydropyrimidines 4a-i}

Of the nine THPs $\mathbf{4 a - i}$ tested, only $\mathbf{4 b}, \mathbf{4 e}, \mathbf{4 d}, \mathbf{4} \mathbf{i}$ and $\mathbf{4 h}$ exhibited anti-bacterial effect on the four-gram positive bacteria. None of the compounds inhibited the growth of E. coli, a gramnegative species, at the concentrations tested. Compound $\mathbf{4 b}$ inhibited the growth of $S$. aureus, $S$. epidermidis, B. subtilis, B. mycoides at $10 \mathrm{mg} / \mathrm{ml}$ and $5 \mathrm{mg} / \mathrm{ml}$ concentrations. The differences in the mean zones of inhibitions observed were not significant, when comparisons are made both within a concentration and between the concentrations (Table 1). Compound $\mathbf{4 b}$ had a greater bactericidal effect on B. mycoides than on the other species; growth of $B$. mycoides was affected at $2.5 \mathrm{mg} / \mathrm{ml}$ and $1.25 \mathrm{mg} / \mathrm{ml}$ concentrations of $\mathbf{4 b}$ also. The mean zone of inhibition at these concentrations, however, was not significantly different from the values obtained at higher concentrations. Compound $\mathbf{4 e}$ inhibited the growth of $S$. aureus, S. epidermidis and B. subtilis. This compound was not bactericidal on B. mycoides and $E$. coli at the concentrations tested. The values of the zones of inhibition recorded in $S$. aureus, S. epidermidis and $B$. subtilis cultures were not significantly different at $10 \mathrm{mg} / \mathrm{ml}$ concentration of $4 \mathbf{e}$. This compound inhibited growth of $S$ aureus at $5 \mathrm{mg} / \mathrm{ml}$ concentration and B. subtilis at $5 \mathrm{mg} / \mathrm{ml}, 2.5 \mathrm{mg} / \mathrm{ml}, 1.25 \mathrm{mg} / \mathrm{ml}$ concentrations; however, significant differences did not exist at these concentrations (Table 1). When S. aureus and B. subtilis cultures were exposed to $10 \mathrm{mg} / \mathrm{ml}$ concentration of $4 \mathbf{e}$, the mean 
zone of inhibition observed, $0.68 \pm 0.08 \mathrm{~cm}$ and $0.72 \pm 0.05 \mathrm{~cm}$ respectively were significantly higher than the mean zone of inhibition observed $(0.1 \pm 0.1 \mathrm{~cm})$ when $S$. aureus was exposed to 5 $\mathrm{mg} / \mathrm{ml}$ of $4 \mathbf{e}$.

Compound 4d inhibited the growth of S.aureus, S.epidermidis and B.subtilis only at $10 \mathrm{mg} / \mathrm{ml}$ and $5 \mathrm{mg} / \mathrm{ml}$ concentrations; but significant differences between the mean values did not exist. B. mycoides and E.coli were not affected by $\mathbf{4 d}$.

Compound $4 \mathbf{i}$ inhibited growth of $S$. epidermidis at $10 \mathrm{mg} / \mathrm{ml}$ concentration. Anti-bacterial effect of this compound was not observed on any of the other cultures at the concentrations tested (Table 1).

Compound $\mathbf{4 h}$ has inhibitory effect on $S$. epidermidis, $S$. aureus, and B. mycoides of which $S$. epidermidis was the least affected; it was inhibited only at $10 \mathrm{mg} / \mathrm{ml}$ concentration. Growth of $S$. aureus, and B. mycoides was inhibited at 10,5 and $2.5 \mathrm{mg} / \mathrm{ml}$ concentrations. The diameter $(0.13 \pm 0.13 \mathrm{~cm})$ of the mean zone of inhibition recorded in $S$. aureus cultures at $2.5 \mathrm{mg} / \mathrm{ml}$ concentration was significantly lower than values recorded due to $\mathbf{4 h}$ except when $S$. aureus was exposed to $5 \mathrm{mg} / \mathrm{ml}$ concentration $(0.64 \pm 0.16 \mathrm{~cm})$.

The anti-bacterial effect of rifampicin was significantly higher on all bacterial species at 1 $\mathrm{mg} / \mathrm{ml}$ concentration when comparisons are made with the anti-bactericidal effect of $\mathbf{4 a - i}$ (Table 1). The solvent controls did not display growth inhibition in any of the bacterial species.

\section{Conclusions}

In conclusion, we have synthesized a set of nine hitherto unknown 5-isonicotinyl-1,2,3,4tetrahydropyrimidines and another set of twelve bis-(5-isonicotinyl-1,2,3,4tetrahydropyrimidines) in good yields. Anti-bacterial properties of the tetrahydropyrimidines have been studied. Compounds $\mathbf{4 b}, \mathbf{4 e}, \mathbf{4 d}, \mathbf{4 i} \& \mathbf{4 h}$ were found to possess anti-bacterial activity on four gram-positive bacteria used in the study. However, variations in anti-bacterial activity, as indicated by mean zones of inhibition, were observed. Compound $\mathbf{4 h}$ produced highest mean zone of inhibition $(1.04 \pm 0.1 \mathrm{~cm})$ on Bacillus mycoides, at $10 \mathrm{mg} / \mathrm{mL}$ concentration

\section{Experimental Section}

General. Melting points were recorded by open capillary method and are uncorrected. The IR spectra were recorded on a Perkin-Elmer 983 spectrometer. ${ }^{1} \mathrm{H}$ NMR and ${ }^{13} \mathrm{C}$ NMR spectra were recorded on Bruker ACF-300 spectrometer. The chemical shifts ( $\delta$ ppm) and the coupling constants $(\mathrm{Hz})$ are reported in the standard fashion with reference to TMS as internal reference. FAB-mass spectra (MS) were measured on JEOL 3SX 102/DA-6000 mass spectrometer using argon as the carrier and m-nitrobenzylalcohol as the matrix. Elemental analyses were performed on a Vario-EL III instrument. Enaminone 2 was synthesized by a reported procedure. ${ }^{16}$ 
3-Phenylamino-1-pyridin-4-ylpropenone 3a. To a solution of enaminone 2 (1 mmol) in $2 \mathrm{~mL}$ acetic acid was added aniline $(1 \mathrm{mmol})$ and the resulting mixture was stirred at room temperature when a solid product precipitated out. After the completion of the reaction (tlc, 45 hours), the mixture was poured over chilled water and the precipitated product was collected by filtration, washed repeatedly by water to ensure complete removal of acid and dried to give practically pure 3a in $82 \%$ yields. It was recrystallised from hexane-ethyl acetate.

3a was obtained as a pale yellow solid, $\mathrm{mp} 149-150{ }^{0} \mathrm{C}$, ir (KBr): 1566, 1639, 3032, $3217 \mathrm{~cm}^{-1}$; ${ }^{1} \mathrm{H} \mathrm{nmr}\left(\mathrm{CDCl}_{3}\right): \delta 6.00(\mathrm{~d}, 1 \mathrm{H}, J=6 \mathrm{~Hz}), 7.13-7.16(\mathrm{~m}, 3 \mathrm{H}), 7.36-7.41(\mathrm{~m}, 2 \mathrm{H}), 7.61(\mathrm{dd}, 1 \mathrm{H}$, $6 \mathrm{~Hz}, 12 \mathrm{~Hz}), 7.73$ (d, 2H, 6Hz), 8.76 (d, 2H, 6Hz), 12.25 (d, 1H, 12Hz); MS: m/z $224\left(\mathrm{M}^{+}\right), 225$ $\left(\mathrm{MH}^{+}\right)$. Anal. Calcd for $\mathrm{C}_{14} \mathrm{H}_{12} \mathrm{~N}_{2} \mathrm{O}: \mathrm{C}, 74.98 ; \mathrm{H}, 5.39 ; \mathrm{N}, 12.49$. Found: C, 74.75; H, 5.50; N, $12.42 \%$.

3-Benzylamino-1-pyridin-4-ylpropenone 3b. To a solution of enaminone 2 (1 mmol) in $3 \mathrm{ml}$ ethanol was added benzylamine $(1.2 \mathrm{mmol})$ and the resulting mixture was refluxed. After the completion of the reaction (tlc, 48 hours), ethanol was distilled off to give a gum, which on trituration with hexane yielded practically pure $\mathbf{3 b}$ in $61 \%$ yield. It was recrystallised from hexane-ethylacetate.

3b was obtained as a light brown solid, mp $113-115{ }^{0} \mathrm{C}$ ir $(\mathrm{KBr}): 1566,1639,3025,3443 \mathrm{~cm}^{-1}$; ${ }^{1} \mathrm{H} \mathrm{nmr}\left(\mathrm{CDCl}_{3}\right): \delta 4.49(\mathrm{~d}, 2 \mathrm{H}, J=6 \mathrm{~Hz}), 5.74(\mathrm{~d}, 1 \mathrm{H}, J=6 \mathrm{~Hz}), 7.10(\mathrm{dd}, 1 \mathrm{H}, J=6 \mathrm{~Hz}, 12.9 \mathrm{~Hz})$, 7.27-7.39 (m, 5H), $7.66(\mathrm{~d}, 2 \mathrm{H}, J=5.7), 8.68(\mathrm{~d}, 2 \mathrm{H}, J=5.7), 10.76$ (br s, $1 \mathrm{H}) ;{ }^{13} \mathrm{C} \mathrm{nmr}\left(\mathrm{CDCl}_{3}\right): \delta$ 52.9, 90.6, 120.7, 127.3, 128.0, 128.9, 137.1, 146.1, 150.3, 155.2, 187.7; MS: m/z $238\left(\mathrm{M}^{+}\right), 239$ $\left(\mathrm{MH}^{+}\right)$. Anal. Calcd for $\mathrm{C}_{15} \mathrm{H}_{14} \mathrm{~N}_{2} \mathrm{O}: \mathrm{C}, 75.61 ; \mathrm{H}, 5.92 ; \mathrm{N}, 11.76$. Found: C, 75.85; H, 6.01; N, $11.82 \%$.

3-Methylamino-1-pyridin-4-ylpropenone 3c. To a solution of enaminone 2 (1 mmol) in $3 \mathrm{ml}$ ethanol was added an aqueous solution of methylamine ( $3 \mathrm{mmol}, 40 \%$ solution) and the resulting mixture was stirred at $50^{\circ} \mathrm{C}$. After the completion of the reaction (tlc, 40 hours), ethanol was distilled off to give a brown gum, which was dissolved in chloroform $(3 \mathrm{~mL})$. This solution was washed with water (2X $2 \mathrm{~mL})$, dried over anhydrous $\mathrm{Na}_{2} \mathrm{SO}_{4}$ and chloroform distilled off to give the product in $52 \%$ yields. It was further purified by column chromatography (silica gel, 20\% EtOAc-hexane).

3c was obtained as light brown gum, ir (KBr): 1527, 1566, 1639, 3005, $3244 \mathrm{~cm}^{-1} ;{ }^{1} \mathrm{H} \mathrm{nmr}$ $\left(\mathrm{CDCl}_{3}\right): \delta 3.15(\mathrm{~d}, 3 \mathrm{H}, J=6 \mathrm{~Hz}), 5.90(\mathrm{~d}, 1 \mathrm{H}, J=7.5 \mathrm{~Hz}), 7.30(\mathrm{dd}, 1 \mathrm{H}, J=7.5,12 \mathrm{~Hz}), 7.95(\mathrm{~d}, 2 \mathrm{H}$, $J=6 \mathrm{~Hz}), 9.10(\mathrm{~d}, 2 \mathrm{H}, J=6 \mathrm{~Hz}), 11.01(\mathrm{~m}, 1 \mathrm{H})$. Anal. Calcd for $\mathrm{C}_{9} \mathrm{H}_{10} \mathrm{~N}_{2} \mathrm{O}: \mathrm{C}, 66.65 ; \mathrm{H}, 6.21 ; \mathrm{N}$, 17.27. Found: C, 66.51; H, 6.28; N, 17.36\%.

\section{(1,2,3,4-Tetrahydro-1,3-disubstitutedpyrimidin-5-yl)(pyridin-4-yl)methanone 4a-i. General procedure}

A mixture of primary amine ( $1 \mathrm{mmol})$, formaldehyde $(2 \mathrm{mmol}, 40 \%$ solution $)$ in $1 \mathrm{~mL}$ methanol was stirred at room temperature for 5-10 minutes. To this was added a solution of $\mathbf{3}$ (1 mmol) in $3 \mathrm{~mL}$ of methanol and the resulting mixture was refluxed. At the end of the reaction (5-28 hours, monitored by tlc), methanol was removed under reduced pressure. The residue was dissolved in 
chloroform $(3 \mathrm{~mL})$, the solution washed with water $(2 \mathrm{X} 2 \mathrm{~mL})$, dried over anhydrous $\mathrm{Na}_{2} \mathrm{SO}_{4}$ and the solvent distilled off to give a gum. The gum was triturated with hexane (4a-c,e,f) to give practically pure product or was subjected to column chromatography (4d,g-i) using silica gel and EtOAc as eluent. 4a-c,e-f were further purified by crystallization from EtOAc-hexane.

(1,2,3,4-Tetrahydro-3-methyl-1-phenylpyrimidin-5-yl)(pyridin-4-yl)methanone 4a. Pale yellow solid, yield: $80 \%$, mp $115-117{ }^{0} \mathrm{C}$; ir (KBr): 1493, $1573 \mathrm{~cm}^{-1} ;{ }^{1} \mathrm{H} \mathrm{nmr}\left(\mathrm{CDCl}_{3}\right): \delta 2.53(\mathrm{~s}$, $3 \mathrm{H}), 3.77(\mathrm{~s}, 2 \mathrm{H}), 4.50(\mathrm{~s}, 2 \mathrm{H}), 6.95(\mathrm{~d}, 2 \mathrm{H}), 7.14-7.18(\mathrm{~m}, 1 \mathrm{H}), 7.33-7.46(\mathrm{~m}, 5 \mathrm{H}), 8.69(\mathrm{~d}, 2 \mathrm{H})$; ${ }^{13} \mathrm{C} \mathrm{nmr}\left(\mathrm{CDCl}_{3}\right): \delta 41.0,49.1,69.7,108.8,118.8,122.3,124.9,129.8,144.1,145.6,146.9$, 150.0, 191.2; MS: m/z $280\left(\mathrm{MH}^{+}\right)$.

Anal. Calcd for $\mathrm{C}_{17} \mathrm{H}_{17} \mathrm{~N}_{3} \mathrm{O}$ : C, 73.10; H, 6.13, N, 15.04. Found: C, 73.31; H, 6.19; N, 15.11\%.

(1,2,3,4-Tetrahydro-3-benzyl-1-phenylpyrimidin-5-yl)(pyridin-4-yl)methanone $4 \mathrm{~b}$. Pale yellow solid, yield: $78 \%$, mp 139-140 ${ }^{0} \mathrm{C}$; ir $(\mathrm{KBr}): 1500,1600 \mathrm{~cm}^{-1} ;{ }^{1} \mathrm{H} \mathrm{nmr}\left(\mathrm{CDCl}_{3}\right): \delta 3.77(\mathrm{~s}$, 2H), $3.90(\mathrm{~s}, 2 \mathrm{H}), 4.51(\mathrm{~s}, 2 \mathrm{H}), 6.88(\mathrm{~d}, 2 \mathrm{H}), 7.11-7.16(\mathrm{~m}, 1 \mathrm{H}), 7.27-7.35(\mathrm{~m}, 7 \mathrm{H}), 7.40-7.42$ (m, 2H), 7.42-7.51 (m, 1H), $8.69(\mathrm{~d}, 2 \mathrm{H}) ;{ }^{13} \mathrm{C} \mathrm{nmr}\left(\mathrm{CDCl}_{3}\right): \delta 47.1,57.0,67.0,108.9,118.7,122.3$, 124.8, 127.6, 128.4, 129.0, 129.7, 137.3, 144.0, 146.0, 146.9, 150.0, 191.2; MS: m/z $355\left(\mathrm{M}^{+}\right)$, $356 \mathrm{MH}^{+}$). Anal. Calcd for $\mathrm{C}_{23} \mathrm{H}_{21} \mathrm{~N}_{3} \mathrm{O}: \mathrm{C}, 77.72 ; \mathrm{H}, 5.96, \mathrm{~N}, 11.82$. Found: $\mathrm{C}, 77.50 ; \mathrm{H}, 5.91 ; \mathrm{N}$, $11.91 \%$.

(1,2,3,4-Tetrahydro-1,3-diphenylpyrimidin-5-yl)(pyridin-4-yl)methanone 4c. Pale yellow solid, yield: 80\%, mp 167-168 ${ }^{0} \mathrm{C}$; ir (KBr): 1500, $1573 \mathrm{~cm}^{-1} ;{ }^{1} \mathrm{H} \mathrm{nmr}\left(\mathrm{CDCl}_{3}\right): \delta 4.49$ (s, 2H), $5.17(\mathrm{~s}, 2 \mathrm{H}), 6.90-6.98(\mathrm{~m}, 5 \mathrm{H}), 7.15-7.27(\mathrm{~m}, 3 \mathrm{H}), 7.33-7.42(\mathrm{~m}, 5 \mathrm{H}), 8.67(\mathrm{~d}, 2 \mathrm{H}) ;{ }^{13} \mathrm{C} \mathrm{nmr}$ $\left(\mathrm{CDCl}_{3}\right): \delta 46.6,65.7,110.2,117.8,118.8,121.4,122.3,125.1,129.3,129.9,143.5,146.6$, 146.7, 148.1, 149.9, 150.3, 190.6; MS: m/z $341\left(\mathrm{M}^{+}\right), 342\left(\mathrm{MH}^{+}\right)$. Anal. Calcd for $\mathrm{C}_{22} \mathrm{H}_{19} \mathrm{~N}_{3} \mathrm{O}: \mathrm{C}$, 77.40; H, 5.61, N, 12.31. Found: C, 77.23; H, 5.68; N, 12.25\%.

(1,2,3,4-Tetrahydro-1-benzyl-3-methylpyrimidin-5-yl)(pyridin-4-yl)methanone 4d. Light brown gum, yield: $63 \%$, ir (KBr): $1577,1633 \mathrm{~cm}^{-1} ;{ }^{1} \mathrm{H} \mathrm{nmr}\left(\mathrm{CDCl}_{3}\right): \delta 2.38(\mathrm{~s}, 3 \mathrm{H}), 3.61(\mathrm{~s}, 2 \mathrm{H})$, 3.89 (s, 2H), 4.33 (s, 2H), 7.15-7.38 (m, 8H), 8.66 (d, 2H); MS: m/z $293\left(\mathrm{M}^{+}\right), 294\left(\mathrm{MH}^{+}\right)$. Anal. Calcd for $\mathrm{C}_{18} \mathrm{H}_{19} \mathrm{~N}_{3} \mathrm{O}$ : C, 73.69; H, 6.53; N, 14.32. Found: C, 73.59; H, 6.48; N, 14.41\%.

(1,2,3,4-Tetrahydro-1,3-dibenzylpyrimidin-5-yl)(pyridin-4-yl)methanone 4e. Light brown solid, yield: $60 \%, \mathrm{mp} 129-130{ }^{0} \mathrm{C}$; ir $(\mathrm{KBr}): 1567,1630 \mathrm{~cm}^{-1} ;{ }^{1} \mathrm{H} \mathrm{nmr}\left(\mathrm{CDCl}_{3}\right): \delta 3.61(\mathrm{~s}, 2 \mathrm{H})$, 3.75 (s, 2H), $3.92(\mathrm{~s}, 2 \mathrm{H}), 4.22(\mathrm{~s}, 2 \mathrm{H}), 7.13-7.21(\mathrm{~m}, 8 \mathrm{H}), 7.25-7.36(\mathrm{~m}, 5 \mathrm{H}), 8.66(\mathrm{~d}, 2 \mathrm{H}) ;{ }^{13} \mathrm{C}$ $\mathrm{nmr}\left(\mathrm{CDCl}_{3}\right): \delta 47.5,57.4,58.2,65.8,105.3,122.4,127.4,127.6,128.4,129.0,135.2,137.5$, 147.4, 149.8, 150.5, 189.8; MS: m/z $369\left(\mathrm{M}^{+}\right), 370\left(\mathrm{MH}^{+}\right)$. Anal. Calcd for $\mathrm{C}_{24} \mathrm{H}_{23} \mathrm{~N}_{3} \mathrm{O}: \mathrm{C}, 78.02$; H, 6.27; N, 11.37. Found: C, 78.22; H, 6.21; N, 11.45\%.

(1,2,3,4-Tetrahydro-1-benzyl-3-phenylpyrimidin-5-yl)(pyridin-4-yl)methanone $4 \mathrm{f}$. Light brown solid, yield: $58 \%$, mp $149-151{ }^{0} \mathrm{C}$; ir (KBr): 1564, 1615; ${ }^{1} \mathrm{H} \mathrm{nmr}\left(\mathrm{CDCl}_{3}\right): \delta 4.30(\mathrm{~s}, 2 \mathrm{H})$, $4.34(\mathrm{~s}, 2 \mathrm{H}), 4.60(\mathrm{~s}, 2 \mathrm{H}), 6.85-6.94(\mathrm{~m}, 3 \mathrm{H}), 7.07-7.32(\mathrm{~m}, 5 \mathrm{H}), 7.34-7.40(\mathrm{~m}, 5 \mathrm{H}), 8.65(\mathrm{~d}$, $2 \mathrm{H}) ;{ }^{13} \mathrm{C} \mathrm{nmr} \delta 45.1,58.2,65.3,106.5,117.8,121.2,122.4,127.5,128.4,129.1,129.3,134.8$, 147.2, 148.2, 149.9, 151.3, 189.4; MS: m/z $355\left(\mathrm{M}^{+}\right), 356\left(\mathrm{MH}^{+}\right)$. Anal. Calcd for $\mathrm{C}_{23} \mathrm{H}_{21} \mathrm{~N}_{3} \mathrm{O}: \mathrm{C}$, 77.72; H, 5.96; N, 11.82. Found: C, 77.95; H, 5.89; N, 11.78\%. 
(1,2,3,4-Tetrahydro-1,3-dimethylpyrimidin-5-yl)(pyridin-4-yl)methanone 4g. Light brown gum, yield: 53\%, ir (KBr): 1560, $1619 \mathrm{~cm}^{-1} ;{ }^{1} \mathrm{H} \mathrm{nmr}\left(\mathrm{CDCl}_{3}\right): \delta 2.48$ (s, 3H), 2.97 (s, 3H), 3.91 $(\mathrm{s}, 2 \mathrm{H}), 4.36(\mathrm{~s}, 2 \mathrm{H}), 6.97(\mathrm{~s}, 1 \mathrm{H}), 7.32(\mathrm{~d}, 2 \mathrm{H}) 8.65(\mathrm{~d}, 2 \mathrm{H}) ;{ }^{13} \mathrm{C} \mathrm{nmr}\left(\mathrm{CDCl}_{3}\right): \delta$ 41.33, 41.50, 70.23, 105.10, 122.50, 147.76, 149.85, 150.26, 159.56, 190.2; MS: m/z $217\left(\mathrm{M}^{+}\right), 218\left(\mathrm{MH}^{+}\right)$. Anal. Calcd for $\mathrm{C}_{12} \mathrm{H}_{15} \mathrm{~N}_{3} \mathrm{O}: \mathrm{C}, 66.34 ; \mathrm{H}, 6.96 ; \mathrm{N}, 19.34$. Found: C, 66.51; H, 6.90; N, 13.41\%.

(1,2,3,4-Tetrahydro-3-benzyl-1-methlpyrimidin-5-yl)(pyridin-4-yl)methanone $4 \mathrm{~h}$. Light brown gum, yield: $46 \%$, ir (KBr): 1619,1626. $\mathrm{cm}^{-1} ;{ }^{1} \mathrm{H} \mathrm{nmr}\left(\mathrm{CDCl}_{3}\right): \delta 2.80(\mathrm{~s}, 3 \mathrm{H}), 3.61(\mathrm{~s}, 2 \mathrm{H})$, $3.65(\mathrm{~s}, 2 \mathrm{H}), 3.82(\mathrm{~s}, 2 \mathrm{H}), 6.92(\mathrm{~s}, 1 \mathrm{H}), 7.16-7.26(\mathrm{~m}, 7 \mathrm{H}), 8.55(\mathrm{~d}, 2 \mathrm{H}) ;{ }^{13} \mathrm{C} \mathrm{nmr}\left(\mathrm{CDCl}_{3}\right): \delta 40.6$, 46.12, 56.42, 66.21, 121.41, 126.0, 126.62, 127.19, 127.55, 127.87, 128.06, 148.71, 150.34, 188.40. Anal. Calcd for $\mathrm{C}_{18} \mathrm{H}_{19} \mathrm{~N}_{3} \mathrm{O}: \mathrm{C}, 73.69 ; \mathrm{H}, 6.53 ; \mathrm{N}, 14.32$. Found: C, 73.91; H, 6.45; N, $14.42 \%$.

(1,2,3,4-Tetrahydro-1-methyl-3-phenylpyrimidin-5-yl)(pyridin-4-yl)methanone 4i. Light brown gum, yield: $80 \%$, ir (KBr): $1546,1593 \mathrm{~cm}^{-1} ;{ }^{1} \mathrm{H} \mathrm{nmr}\left(\mathrm{CDCl}_{3}\right): \delta 2.99(\mathrm{~s}, 3 \mathrm{H}), 4.33(\mathrm{~s}, 2 \mathrm{H})$, $4.63(\mathrm{~s}, 2 \mathrm{H}), 6.92-7.02(\mathrm{~m}, 4 \mathrm{H}), 7.26-7.32(\mathrm{~m}, 4 \mathrm{H}) 8.64(\mathrm{~d}, 2 \mathrm{H}) .{ }^{13} \mathrm{C} \mathrm{nmr}\left(\mathrm{CDCl}_{3}\right):$ 828.68, 40.09, 44.14, 65.53, 105.06, 116.77, 121.40, 121.66, 128.38, 147.47, 148.75, 150.91, 188.03. Anal. Calcd for $\mathrm{C}_{17} \mathrm{H}_{17} \mathrm{~N}_{3} \mathrm{O}: \mathrm{C}, 73.10 ; \mathrm{H}, 6.13 ; \mathrm{N}, 15.04$. Found: C, 73.26; H, 6.06; N, 15.11\%.

\section{Synthesis of bis-tetrahydropyrimidines. General procedure}

A mixture of diamine $(0.5 \mathrm{mmol})$ and formaldehyde $(2 \mathrm{mmol})$ in $1 \mathrm{~mL}$ of methanol was stirred at room temperature for 5-10 minutes. To this was added a solution of the enaminone 3 ( $1 \mathrm{mmol}$ ) in $4 \mathrm{~mL}$ of methanol and the resulting solution was refluxed for 4-12 hours. On completion of the reaction (tlc), the solvent was distilled off. The residue was dissolved in $3 \mathrm{~mL}$ of chloroform and the solution washed with water $(2 \mathrm{X} 2 \mathrm{~mL})$, dried $\left(\mathrm{Na}_{2} \mathrm{SO}_{4}\right)$ and chloroform distilled off to give a gum from which the product 4 was isolated by column chromatography (silica gel, EtOAc) in 40-65\% yields.

[3,3'-(Ethane-1,2-diyl)bis(1-phenyl-1,2,3,4-tetrahydropyrimidine-5,3-diyl)]bis (pyridin-4ylmethanone) 5a. White solid, yield 55\%, mp 189-191 ${ }^{0} \mathrm{C}$; ir $(\mathrm{KBr}): 1541,1596,1649 \mathrm{~cm}^{-1} ;{ }^{1} \mathrm{H}$ $\mathrm{nmr}\left(\mathrm{CDCl}_{3}\right): \delta 2.80(\mathrm{~s}, 4 \mathrm{H}), 3.83(\mathrm{~s}, 4 \mathrm{H}), 4.64(\mathrm{~s}, 4 \mathrm{H}), 6.92(\mathrm{~d}, 4 \mathrm{H}), 7.15-7.20(\mathrm{~m}, 2 \mathrm{H}), 7.27-$ $7.51(\mathrm{~m}, 10 \mathrm{H}), 8.69(\mathrm{~d}, 4 \mathrm{H})$; MS: m/z $556\left(\mathrm{M}^{+}\right)$. Anal. Calcd for $\mathrm{C}_{34} \mathrm{H}_{32} \mathrm{~N}_{6} \mathrm{O}_{2}$ : C, 73.36; H, 5.79; N, 15.10. Found: C, 73.55; H, 5.73; N, 15.02\%.

(3,3'-(Propane-1,3-diyl)bis(1-phenyl-1,2,3,4-tetrahydropyrimidine-5,3-diyl))bis(pyridin-4-

ylmethanone) 5b. White solid, yield $65 \%$, mp 193-195 ${ }^{0} \mathrm{C}$; ir $(\mathrm{KBr}): 1495,1568 \mathrm{~cm}^{-1}$; ${ }^{1} \mathrm{H} \mathrm{nmr}$ $\left(\mathrm{CDCl}_{3}\right): \delta 1.75-1.82(\mathrm{~m}, 2 \mathrm{H}), 2.68(\mathrm{t}, 4 \mathrm{H}), 3.78(\mathrm{~s}, 4 \mathrm{H}), 4.54(\mathrm{~s}, 4 \mathrm{H}), 6.93(\mathrm{~d}, 4 \mathrm{H})$ 7.13-7.18 (m, 2H), 7.27-7.50 (m, 10H), $8.69(\mathrm{~d}, 4 \mathrm{H}) ;{ }^{13} \mathrm{C} \mathrm{nmr}\left(\mathrm{CDCl}_{3}\right): 826.3,47.3,50.3,68.4,108.9,118.6$, 122.3, 124.9, 129.8, 144.0, 146.0, 146.8, 150.0, 191.2. Anal. Calcd for $\mathrm{C}_{35} \mathrm{H}_{34} \mathrm{~N}_{6} \mathrm{O}_{2}$ : C, 73.66; H, $6.01 ; \mathrm{N}, 14.73$. Found: C, 73.81; H, 6.09; N, 14.81\%.

(3,3'-(Butane-1,4-diyl)bis(1-phenyl-1,2,3,4-tetrahydropyrimidine-5,3-diyl))bis(pyridin-4-ylmethanone) 5c. Light yellow solid, yield 60\%, mp 153-154 ${ }^{0} \mathrm{C}$; ir $(\mathrm{KBr}): 1498,1568 \mathrm{~cm}^{-1} ;{ }^{1} \mathrm{H}$ $\mathrm{nmr}\left(\mathrm{CDCl}_{3}\right)$ : $\delta 1.60-1.61(\mathrm{~m}, 4 \mathrm{H}), 2.59-2.60(\mathrm{~m}, 4 \mathrm{H}), 3.79(\mathrm{~s}, 4 \mathrm{H}), 4.54(\mathrm{~s}, 4 \mathrm{H}), 6.93(\mathrm{~d}, 4 \mathrm{H})$, 
7.14-7.18 (m, 2H), 7.27-7.45 (m, 10H), $8.69(\mathrm{~d}, 4 \mathrm{H}) ; \mathrm{MS}: \mathrm{m} / \mathrm{z} 585\left(\mathrm{MH}^{+}\right)$. Anal. Calcd for $\mathrm{C}_{36} \mathrm{H}_{36} \mathrm{~N}_{6} \mathrm{O}_{2}$ : C, 73.95; H, 6.21; N, 14.37. Found: C, 73.83; H, 6.25; N, 14.32\%.

(3,3'-(1,4-Phenylene)bis(1-phenyl-1,2,3,4-tetrahydropyrimidine-5,3-diyl))bis(pyridin-4-ylmethanone) 5d. Yellow solid, yield $62 \%$, mp 168-170 ${ }^{0} \mathrm{C}$; ir $(\mathrm{KBr}): 1563,1619,1655 \mathrm{~cm}^{-1} ;{ }^{1} \mathrm{H}$ $\mathrm{nmr}\left(\mathrm{CDCl}_{3}\right): \delta 4.39(\mathrm{~s}, 4 \mathrm{H}), 5.08(\mathrm{~s}, 4 \mathrm{H}), 6.87-6.93(\mathrm{~m}, 4 \mathrm{H}), 7.15-7.46(\mathrm{~m}, 16 \mathrm{H}), 8.68(\mathrm{~d}, 4 \mathrm{H})$; MS: $\mathrm{m} / \mathrm{z} 605\left(\mathrm{MH}^{+}\right)$. Anal. Calcd for $\mathrm{C}_{38} \mathrm{H}_{32} \mathrm{~N}_{6} \mathrm{O}_{2}$ : C, 75.48; H, 5.33; N, 13.90. Found: C, 75.31; H, 5.38; N, 13.84\%.

(3,3'-(Ethane-1,2-diyl)bis(1-benzyl-1,2,3,4-tetrahydropyrimidine-5,3-diyl))bis(pyridin-4-ylmethanone) 5e. Light brown gum, yield 45\%, ir (KBr): $1561,1656 \mathrm{~cm}^{-1} ;{ }^{1} \mathrm{H} \mathrm{nmr}\left(\mathrm{CDCl}_{3}\right): \delta 2.55$ $(\mathrm{s}, 4 \mathrm{H}), 3.63(\mathrm{~s}, 4 \mathrm{H}), 3.98(\mathrm{~s}, 4 \mathrm{H}), 4.27(\mathrm{~s}, 4 \mathrm{H}), 7.18-7.32(\mathrm{~m}, 16 \mathrm{H}), 8.65(\mathrm{~d}, 4 \mathrm{H}) ;{ }^{13} \mathrm{C} \mathrm{nmr}$ $\left(\mathrm{CDCl}_{3}\right)$ : $\delta 29.5,46.9,50.5,58.2,67.3,104.9,121.0,122.3,127.6,128.4,129.0,147.3,150.5$, 189.7. Anal. Calcd for $\mathrm{C}_{36} \mathrm{H}_{36} \mathrm{~N}_{6} \mathrm{O}_{2}$ : C, 73.95; H, 6.21; N, 14.37. Found: C, 73.99; H, 6.26; N, $14.41 \%$.

(3,3'-(Propane-1,3-diyl)bis(1-benzyl-1,2,3,4-tetrahydropyrimidine-5,3-diyl))bis(pyridin-4ylmethanone) 5f. Light brown gum, yield 45\%, ir (KBr): 1563, $1656 \mathrm{~cm}^{-1} ;{ }^{1} \mathrm{H} \mathrm{nmr}\left(\mathrm{CDCl}_{3}\right)$; 81.42-1.52 (m, 2H), $2.40(\mathrm{t}, 4 \mathrm{H}), 3.61(\mathrm{~s}, 4 \mathrm{H}), 3.86(\mathrm{~s}, 4 \mathrm{H}), 4.27(\mathrm{~s}, 4 \mathrm{H})$ 7.17-7.19 (m, 6H), 7.32$7.37(\mathrm{~m}, 10 \mathrm{H}), 8.63(\mathrm{~d}, 4 \mathrm{H}) ;{ }^{13} \mathrm{C} \mathrm{nmr}\left(\mathrm{CDCl}_{3}\right): \delta 25.6,47.0,50.4,51.8,58.1,66.4,105.0,121.0$, 122.2, 127.4, 128.5, 128.9, 147.3, 149.6, 189.5. Anal. Calcd for $\mathrm{C}_{37} \mathrm{H}_{38} \mathrm{~N}_{6} \mathrm{O}_{2}$ : C, 74.22; H, 6.40; N, 14.04. Found: C, 74.05; H, 6.43; N, 14.11\%.

(3,3'-(Butane-1,4-diyl)bis(1-benzyl-1,2,3,4-tetrahydropyrimidine-5,3-diyl))bis(pyridin-4-ylmethanone) 5g. Light brown gum, yield 50\%, ir (KBr): 1563, $1659 \mathrm{~cm}^{-1} ;{ }^{1} \mathrm{H} \mathrm{nmr}\left(\mathrm{CDCl}_{3}\right)$ : 81.25-1.33 (m, 4H), 2.34-2.41 (m, 4H), $3.64(\mathrm{~s}, 4 \mathrm{H}), 3.93(\mathrm{~s}, 4 \mathrm{H}), 4.27(\mathrm{~s}, 4 \mathrm{H})$ 7.15-7.35 (m, $16 \mathrm{H}), 8.67$ (d, 4H); MS: m/z $612\left(\mathrm{M}^{+}\right)$. Anal. Calcd for $\mathrm{C}_{38} \mathrm{H}_{40} \mathrm{~N}_{6} \mathrm{O}_{2}:$ C, 74.48; H, 6.58; N, 13.71 . Found: C, 74.31; H, 6.54; N, 13.77\%.

(3,3'-(1,4-Phenylene)bis(1-benzyl-1,2,3,4-tetrahydropyrimidine-5,3-diyl))bis(pyridin-4-ylmethanone) 5h. Brown solid, yield 40\%, mp188-190 ${ }^{0} \mathrm{C}$; ir (KBr): 1566, $1654 \mathrm{~cm}^{-1} ;{ }^{1} \mathrm{H} \mathrm{nmr}$ $\left(\mathrm{CDCl}_{3}\right): \delta 4.27(\mathrm{~s} 4 \mathrm{H}), 4.30(\mathrm{~s}, 4 \mathrm{H}), 4.52(\mathrm{~s}, 4 \mathrm{H}), 6.74(\mathrm{~s}, 4 \mathrm{H}), 7.05-7.15(\mathrm{~m}, 6 \mathrm{H}), 7.21-7.35(\mathrm{~m}$, $10 \mathrm{H}), 8.66(\mathrm{~d}, 4 \mathrm{H}) ; \mathrm{MS}: \mathrm{m} / \mathrm{z} 632\left(\mathrm{M}^{+}\right), 633\left(\mathrm{MH}^{+}\right)$. Anal. Calcd for $\mathrm{C}_{40} \mathrm{H}_{36} \mathrm{~N}_{6} \mathrm{O}_{2}$ : C, 75.93; H, 5.73; N, 13.28. Found: C, 75.72; H, 5.69; N, 11.32\%.

(3,3'-(Ethane-1,2-diyl)bis(1-methyl-1,2,3,4-tetrahydropyrimidine-5,3-diyl))bis(pyridin-4ylmethanone) 5i. Light brown gum, yield 45\%, ir (KBr): 1565, $1654 \mathrm{~cm}^{-1} ;{ }^{1} \mathrm{H} \mathrm{nmr}\left(\mathrm{CDCl}_{3}\right)$ : $\delta 2.64(\mathrm{~s}, 4 \mathrm{H}), 3.02(\mathrm{~s}, 6 \mathrm{H}), 3.35(\mathrm{~s}, 4 \mathrm{H}), 3.41(\mathrm{~s}, 4 \mathrm{H}), 6.85(\mathrm{~s}, 2 \mathrm{H}), 7.40(\mathrm{~d}, 4 \mathrm{H}), 8.66(\mathrm{~d}, 4 \mathrm{H})$; ${ }^{13} \mathrm{C} \mathrm{nmr}\left(\mathrm{CDCl}_{3}\right): \delta 40.00,45.11,53.52,70.05,84.82,106.25,122.36,149.60,151.50,189.93$. Anal. Calcd for $\mathrm{C}_{24} \mathrm{H}_{28} \mathrm{~N}_{6} \mathrm{O}_{2}$ : C, 66.65; H, 6.53; N, 19.43. Found: C, 66.81; H, 6.57; N, 19.50\%. (3,3'-(Propane-1,3-diyl)bis(1-methyl-1,2,3,4-tetrahydropyrimidine-5,3-diyl))bis(pyridin-4ylmethanone) (5j). Light brown gum, yield 55\%, ir (KBr): 1560, $1655 \mathrm{~cm}^{-1} ;{ }^{1} \mathrm{H} \mathrm{nmr}\left(\mathrm{CDCl}_{3}\right)$ : 81.74-1.78 (m, 2H), $2.64(\mathrm{t}, 4 \mathrm{H}), 2.98(\mathrm{~s}, 6 \mathrm{H}), 3.31(\mathrm{~s}, 4 \mathrm{H}), 3.48(\mathrm{~s}, 4 \mathrm{H}) 6.97(\mathrm{~s}, 2 \mathrm{H}), 7.33(\mathrm{~d}$, $4 \mathrm{H}), 8.66(\mathrm{~d}, 4 \mathrm{H}) ;{ }^{13} \mathrm{C} \mathrm{nmr}\left(\mathrm{CDCl}_{3}\right): \delta 26.13,41.21,46.53,50.95,68.86,104.95,122.33,149.63$, 150.25, 151.20, 189.20. Anal. Calcd for $\mathrm{C}_{25} \mathrm{H}_{30} \mathrm{~N}_{6} \mathrm{O}_{2}$ : C, 67.24; H, 6.77; N, 18.82. Found: C, $67.15 ; \mathrm{H}, 6.75 ; \mathrm{N}, 18.91 \%$. 
(3,3'-(Butane-1,4-diyl)bis(1-methyl-1,2,3,4-tetrahydropyrimidine-5,3-diyl))bis(pyridin-4-yl-

methanone) 5k. Light brown gum, yield $60 \%$, ir $(\mathrm{KBr}): 1563,1655 \mathrm{~cm}^{-1} ;{ }^{1} \mathrm{H} \mathrm{nmr}\left(\mathrm{CDCl}_{3}\right)$ : 81.58-1.73 (m 4H), 2.38-2.57 (m, 4H), $2.96(\mathrm{~s}, 6 \mathrm{H}), 3.74(\mathrm{~s}, 4 \mathrm{H}), 3.95(\mathrm{~s}, 4 \mathrm{H}), 6.96(\mathrm{~s}, 2 \mathrm{H}), 7.32$ (d, $4 \mathrm{H}), 8.70(\mathrm{~d}, 4 \mathrm{H}) ; \mathrm{MS}: \mathrm{m} / \mathrm{z} 461\left(\mathrm{MH}^{+}\right)$. Anal. Calcd for $\mathrm{C}_{26} \mathrm{H}_{32} \mathrm{~N}_{6} \mathrm{O}_{2}: \mathrm{C}, 67.80 ; \mathrm{H}, 7.00 ; \mathrm{N}$, 18.25. Found: C, 67.98; H, 7.06; N, 18.21\%.

(3,3'-(1,4-Phenylene)bis(1-methyl-1,2,3,4-tetrahydropyrimidine-5,3-diyl))bis(pyridin-4-ylmethanone) 5l. Light brown solid, yield $40 \%, \mathrm{mp} 235{ }^{\circ} \mathrm{C}$; ir (KBr): $1615,1654 \mathrm{~cm}^{-1} ;{ }^{1} \mathrm{H} \mathrm{nmr}$ $\left(\mathrm{CDCl}_{3}\right): \delta 3.01(\mathrm{~s} 6 \mathrm{H}), 4.25$ (s, 4H), 4.59 (s, 4H), 6.95-6.99 (m, 6H), 7.32-7.33 (m, 4H), 8.64$8.70(\mathrm{~m}, 4 \mathrm{H}) .{ }^{13} \mathrm{C} \mathrm{nmr}\left(\mathrm{CDCl}_{3}\right): 840.7,45.1,66.6,105.2,118.8,121.9,142.6,146.9,149.4$, 151.7, 188.40. Anal. Calcd for $\mathrm{C}_{28} \mathrm{H}_{28} \mathrm{~N}_{6} \mathrm{O}_{2}$ : C, 69.98; H, 5.87; N, 17.49. Found: C, 69.85; H, $5.90 ; \mathrm{N}, 17.61 \%$.

\section{Anti-bacterial assay}

Lyophilized cultures of the bacterial species (Table 1) were procured from the microbial type culture collection (MTCC) of the Institute of Microbial Technology, Chandigarh, India. Microbial cultures were developed by the Cup Plate Method. ${ }^{18}$ The test molecules, dissolved in the appropriate solvent at $10 \mathrm{mg} / \mathrm{ml}, 5 \mathrm{mg} / \mathrm{ml}, 2.5 \mathrm{mg} / \mathrm{ml}$ and $1.25 \mathrm{mg} / \mathrm{ml}$ concentrations were loaded in the cups. Rifampicin $(1 \mathrm{mg} / \mathrm{ml})$ was loaded in one well as the antibiotic standard. In the plates containing cultures of $B$. mycoides acetone was loaded in one well as the solvent control while in the plates containing other bacterial cultures, DMF was loaded as the solvent control. The volume of material loaded in each well was $10 \mu l$.

Zones of inhibition were considered as indications of anti-bacterial effect of the test compounds and of rifampicin. Observation and measurement of zones of inhibition were done after incubation at $37^{\circ} \mathrm{C}$ for $24 \mathrm{hr}$. The diameter of a zone of inhibition in each replication was recorded and subjected to Analysis of Variance (ANOVA) followed by Tukey's Honestly Significant Difference (Tukey HSD) test to estimate the existence of significant difference between mean zones of inhibition.

\section{Acknowledgements}

The authors wish to thank the Principal, Rev. Fr. Ioannis Warpakma, SDB for the facilities and Rev. Fr. Stephen Mavely, SDB and Rev. Fr. Joseph Nellanatt, SDB for their encouragement during the course of this investigation. The financial support from ICAR-NATP-PIU and UGCNew Delhi is gratefully acknowledged. Authors (KC \& MCD) thank ICAR for Senior Research Fellowships. Thanks are also due to the Heads of RSIC-CDRI (Lucknow) and RSIC-NEHU (Shillong) for recording spectra. 


\section{References}

1. Wu, F.; Kariya, A.; Kumada, H.; Sato, J.; Nanjo, K.; Segami, S.; Takasuka, S.; Henmi, S.; Tsuji, A. Agro-Kanesho Co. Ltd., Jpn. Kokai Tokkyo Koho JP 03264580 (91,264,580) [CL.C07D401/12], 25 Nov 1991, Appl. 90/61,885, 13 March 1990, 7pp; Chem. Abstr. 1992, $116,123318 \mathrm{f}$.

2. Haga, T.; Toki, T.; Koyanagi, T.; Omatsu, M.; Sasaki H.; Morita, M. (Ishihara Sangya Kaisha Ltd.) Jpn. Kokai Tokkyo Koho JP 03200783 (91 200 783) [CL.C07D401/06], 02 Sep 1991, Appl. 89/61,304,372, 28 Dec 1989, 13pp; Chem. Abstr. 1992, 116, 41469s.

3. Karim, E.; Kishore, K.; Vishwakarma, J. N. J. Heterocycl. Chem. 2003, 40, 901.

4. Chanda, K.; Dutta, M. C.; Karim, E.; Vishwakarma, J. N. J. Heterocycl. Chem. 2004, 41, 627.

5. Dutta, M. C.; Chanda, K.; Vishwakarma, J. N. J. Heterocycl. Chem. 2005, 42, 121.

6. Griffin, A. G.; Britt, T. R. J. Am. Chem. Soc. 1981, 103, 4957.

7. Galli, G.; Laus, M.; Angeloni, A. S. Makromol. Chem. 1986, 187, 289.

8. (a) Finkelmann, H. Angew. Chem. 1987, 99, 840. (b) Finkelmann, H. Angew. Chem. (Int. Ed. Engl.) 1987, 26, 816.

9. Aguilera, C.; Parra, M.; Fuentes, G. Z. Naturforsch 1998, 53b, 367.

10. Braun, D.; Langendorf, R. J. Prak. Chem. 1999, 341, 128.

11. Ariens, E. J. In Drug Design; Ariens, E. J., Ed.; Academic Press: New York, 1971; Vol. 1, p 1.

12. Komaraiah, A.; Ramakrishna, K.; Sailu, B.; Reddy, P. S. N. Arkivoc 2007, (xiv), 110.

13. Yachevskii, D. S.; Chizhov, D. L.; Pashkevich, K. I.; Charushin, V. N. Arkivoc 2004, (xi), 71.

14. Jain, M.; Sakhuja, R.; Khanna, P.; Bhagat, S.; Jain, S. C. Arkivoc 2008, (xv), 54.

15. He, Feng-Qi; Liu, Xing-Hai; Wang; Bao-Lei \& Li, Z.-M. Heteroat. Chem. 2008, 19(1), 21.

16. Zhao, L.-X.; Sherchan, J.; Park, J. K.; Jahng, Y.; Jeong, B.-S.; Jeong, T. C.; Le, C.-S.; Lee, E.-S. Arch. Pharm. Res. 2006, 29(12), 1091.

17. Chanda, K.; Dutta, M. C.; Karim, E.; Vishwakarma, J. N. Indian J. Chem. 2004, 43B, 2471.

18. Rose, S. B.; Miller, R. E. J. Bacteriol. 1939, 38, 525. 